\title{
Transición a la adultez en España antes y en la salida de la crisis económica. Una comparación utilizando el análisis de entropia
}

\section{Transition to adulthood, before and at the end of the economic crisis in Spain. A comparison using the entropy analysis}

\author{
F. JAVIER SÁNCHEZ-GALÁN \\ Universidad de Valladolid \\ javiersg@soc.uva.es (ESPAÑA)
}

Recibido: 31.052018

Aceptado: 13.03.2019

\section{RESUMEN}

En este artículo se va a valorar la heterogeneidad en los procesos de transición hacia la vida adulta comparando dos periodos de diferente coyuntura económica: el periodo previo la crisis y en los años en los que es posible considerar como el comienzo de la salida de la crisis económica. A través del índice de entropía se medirá el grado de complejidad, superposición o simultaneidad de los diferentes estatus o estados que estructuran el proceso de transición hacia la vida adulta (estudiante, activo, emancipado, en pareja y ser padres) y atendiendo a diversos factores o variables como la edad, sexo y riesgo de pobreza. Con este indicador será posible construir secuencias donde constatar el final de la transición, situado por la bibliografía especializada en el ápice de entropía o edad a la que se observa la máxima heterogeneidad.

\section{PALABRAS CLAVE}

Transición, heterogeneidad, jóvenes, entropía, crisis económica.

\section{ABSTRACT}

In this article we will assess the heterogeneity in the processes of transition to adult life by comparing two periods of different economic conjuncture: the period prior to the crisis and in the years in which it is possible to consider the 
beginning of the exit from the economic crisis. Through the entropy index, the degree of complexity will be measured, overlap or simultaneity of the different status or states that structure the process of transition to adult life (student, active, emancipated, as a couple and to be parents) and attending to various factors or variables such as age, sex and risk of poverty. With this indicator it will be possible to build sequences to verify the end of the transition, located by specialized bibliography at the apex of entropy or age at which the maximum heterogeneity is observed.

\section{KEY WORDS}

Transition, heterogeneity, youth, entropy, economic crisis.

\section{INTRODUCCIÓN}

En una situación de crisis económica la juventud representa un colectivo especialmente vulnerable en lo que se refiere a afrontar las diferentes transiciones en su ciclo vital: formativa, ocupacional, residencial, relacional y parental; el objetivo general de este artículo es valorar el grado de heterogeneidad, desigualdad y diferenciación de los jóvenes con relación a los procesos de transición hacia la adultez a través de las variables que les definen (estudiar, ser activo, estar emancipado, vivir en pareja y ser padres), entendiendo esta heterogeneidad como la diversidad y multiplicidad en las maneras de afrontar los cambios en los diversos procesos que incluye la transición. Las formas de afrontar estos procesos se ven condicionadas por los componentes estructurales de la sociedad como puede ser la coyuntura económica y variables sociodemográficas como la edad o el sexo.

Nos encontramos en una sociedad globalizada donde las consecuencias de cualquier tipo de catástrofe tiene una dimensión internacional; los riesgos modernos no tienen limitaciones en el espacio y el tiempo, la era de la información y la sociedad tecnológicamente avanzada representan una nueva sociedad, una nueva cultura y una nueva economía al abrigo de las tecnologías de la información, el conocimiento y un nuevo capitalismo (Giddens, 1979; Castells, 1997; Beck, 1998; Tezanos, 2001). La juventud es uno de los colectivos más sensibles al proceso de globalización en las sociedades contemporáneas, la juventud sufre las consecuencias de los grandes movimientos económicos, como la deslocalización de empresas, los procesos migratorios en ambas direcciones, oportunidades de formación y empleo en otros países y la competencia de los inmigrantes en cuanto a empleo, servicios demandados, etc. La juventud es un colectivo especialmente vulnerable en un contexto de crisis económica, tasas de desempleo que duplican a las de la población activa en su conjunto, dificultades para acceder a una vivienda, retraso en la formación de nuevos núcleos familiares, etc., son algunas de las principales circunstancias por las que trascurre la transición de los jóvenes hacia la vida adulta. 
Los procesos de transición hacia la vida adulta de los jóvenes se desarrollan en un continuo: el término del periodo formativo, la incorporación al mercado laboral, la emancipación residencial y la creación de nuevos núcleos familiares pueden considerarse elementos de ese continuo que terminaría con la obtención de la condición de persona adulta. Esta transición no se presenta exenta de dificultades y difiere sustancialmente según el momento histórico y los propios individuos, pero en buena medida se repite la secuencia (Torres y Lapa, 2010; Leccardi, 2010; Toulemon, 2010; Vieira y Miret, 2010).

Los itinerarios y trayectorias estarían definidas por los caminos que recorren los jóvenes hacia la vida adulta a través de los principales elementos de incorporación hacia la adultez, como el sistema educativo, el mercado laboral y la construcción de nuevos núcleos familiares. Esta transición se entiende como un proceso en el que influyen la realidad sociohistórica, las instituciones sociales y la toma de decisiones de los propios jóvenes, y no tener en cuenta todos los factores dificultaría el análisis de los procesos de transición (Furstenberg, 2005; Bird y Kruger, 2005). La realidad sociohistórica y las instituciones sociales estarían representadas por las estructuras sociales que ejercen coerción hacia los individuos, como los modelos políticos y la coyuntura económica, las identidades culturales, el sistema educativo, el mercado de trabajo, etc., mientras que el proceso biográfico o de decisiones propias de los individuos estaría representado por el conjunto de estrategias y toma de decisiones como respuesta a la constricción que ejercen los componentes estructurales. Por tanto los itinerarios y trayectorias tienen un vínculo claro con la estructura y el contexto social, la familia, el entorno, los grupos de iguales, los itinerarios formativos, etc. (Urcola, 2003; Casal et al., 2006; Ulrich, 2008; Vieira y Miret, 2010; Moreno et al., 2012). En definitiva, una perspectiva de transición o itinerario hacia la vida adulta que aglutinaría estructura, acción e historia, un modelo de análisis holista, al que habría que añadir la reversibilidad de los acontecimientos e incluso su no ocurrencia, pues hay quienes viven toda su vida en el hogar de origen, hay quienes nunca se incorporan al mercado laboral, quienes no viven en pareja y quienes nunca son padres o madres (Gentile, 2010; Robette, 2010; Iacovou, 2011; Gaviria, 2017). De esta manera, la transición a la vida adulta en los diferentes ámbitos o procesos, no presenta un modelo único y lineal y cada vez más se dilata en el tiempo.

\section{DIVERSIDAD EN LA TRANSICIÓN HACIA LA VIDA ADULTA}

Una de las características de la transición hacia la vida adulta en España y en el resto de países europeos, es la diversidad en cómo y cuándo se experimentan cada uno de los eventos de transición, en contraste con esa supuesta linealidad que se producía en décadas pasadas. Esta diversidad no tiene por qué ser en si mima un problema, sin embargo algunos autores como Furstenberg (2005) sostienen que los sucesos inoportunos e imprevistos, las secuencias de estatus desordenadas y transiciones no normalizadas tienen consecuencias para el éxito en los itinerarios de transición, en este sentido las transiciones estandarizadas que 
siguen una secuencia normalizada (término de los estudios, inserción laboral, emancipación residencial y creación de nuevos núcleos familiares) regulan el paso a la adultez, crean apoyos sociales, expectativas y recompensas para quienes siguen las pautas culturalmente establecidas. Fustemberg aporta argumentos teóricos que apoyan la idea de que la planificación, el tiempo y la secuencia ordenada de las transiciones aumentan la probabilidad de una transición exitosa. También estudia las vías no normalizadas de transición, las acciones correctivas de los individuos cuando se producen distorsiones en las secuencias normalizadas, como trabajar antes de terminar los estudios, maternidad o divorcio en adolescentes, etc. Más allá de lo deseable o no de una transición normalizada, lo cierto es que la desestandarización o no normalización de las transiciones hacia la adultez parece que es lo que caracterizaría a estos procesos en la juventud europea actual, Du Bois-Reymond y López (2004) hablan de las transiciones tipo yo yo para referirse al orden y grado de reversibilidad de los eventos de transición. Muchos jóvenes experimentan la reversibilidad en sus procesos de transición, es posible estar estudiando y al mismo tiempo trabajando, como también es posible haber experimentado la transición escolar y laboral y retornar de nuevo a los estudios o experimentar la transición residencial y retornar al hogar de origen, etc. La falta de igualdad de oportunidades entre los jóvenes trabajadores británicos, el desajuste entre escuela y mercado laboral entre los italianos o el fracaso de un sistema escolar altamente selectivo entre los jóvenes alemanes que desmotiva a aquellos jóvenes que fracasan en el sistema educativo, serian motivos para trayectorias fallidas que implican reversibilidad en los procesos de transición europeos a juicio de estos autores.

Numerosos estudios valoran la estructura de las transiciones juveniles constatando la precariedad de la juventud en el acceso al trabajo o el retraso continuado de la emancipación residencial, de tal manera que la crisis empeora las ya de por sí condiciones de precariedad de los jóvenes en el acceso al empleo pero que no modifica las pautas de emancipación que se vienen retrasando progresivamente por las dificultades de acceso a empleos bien remunerados, dificultades de acceso a la vivienda y altas tasas de desempleo juvenil. Por otra parte el abandono escolar y la sobre-cualificación son problemas endémicos de España que la crisis económica ha agravado y que dificultan la transición juvenil en su conjunto. Se retrasa la emancipación residencial ante la incertidumbre y poca seguridad en el futuro, asumiendo posturas conservadoras como la prolongación de la vida en la familia de origen donde la juventud goza de un bienestar que temen perder. Estas circunstancias condicionan las trayectorias vitales y las pautas de inserción social (Moreno et al., 2012; Gentile, 2016)

Esta precarización es analizada también en la tesis de Carballo (2014) con especial interés en las estrategias para la emancipación residencial de la juventud en el País Vasco, constatando cómo la precarización en los procesos de transición de la juventud a la adultez tienen clara concordancia con la precarización de la población adulta. Según este autor el proceso de individualización propio de la Modernidad culmina con el acceso a la vivienda en propiedad, cuestión claramente condiciona por la precariedad en los itinerarios de acceso al mercado 
laboral. La precariedad en las trayectorias laborales junto a los desajustes entre formación y empleo son también el objeto de estudio de López-Santamaría (2012) constatando cómo las situaciones laborales de la juventud y las biografías que se van tejiendo tienen un orden complejo repleto de conexiones difícilmente precisas. La inserción laboral juvenil es tratada como un problema de empleabilidad sin considerar la inestabilidad e inseguridad económicas que genera los empleos precarios.

Afrontar las transiciones es siempre un reto cargado de incertidumbres y que pueden acentuar el riesgo de pobreza, los datos apuntan a que los jóvenes en la mayoría de los países europeos se enfrentan a un riesgo de pobreza superior a la media del resto de la población, siendo el factor de mayor riesgo de pobreza entre los jóvenes la emancipación residencial, seguido de no trabajar y tener hijos, mientras el vivir con los padres o compañeros minimiza este riesgo. El nivel de estudios también es un factor predictivo con relación al nivel de pobreza pero sin lugar a dudas es el origen familiar el factor más determinante (Sánchez-Galán y Moreno, 2017). En cualquier caso interesa evidenciar las diferencias que tienen los procesos de transición, en este caso con relación al riesgo de pobreza en los países europeos (Aassve et al., 2005; Fahmy, 2007). En Becker et al. (2005) se ofrecen algunas respuestas con relación a la emancipación tardía en el Sur de Europa y algunas consecuencias de futuro. Estos autores han valorado la influencia que tiene en la emancipación residencial la inseguridad laboral por parte de los padres y por parte de los hijos, constatando que cuando en el hogar de origen los padres sufren inseguridad laboral la emancipación de hijos es más temprana que cuando son los hijos los que perciben inseguridad laboral, en este caso retrasan las salida del hogar. Los efectos de una emancipación residencial tardía serían por una parte una baja movilidad geográfica reduciendo la capacidad de la economía para reaccionar ante incertidumbres económicas y baja fertilidad que pondría en peligro los sistemas de pensiones de los países del Sur de Europa.

Las transiciones hacia la vida adulta son diferentes como lo es la propia estructura social, aun en una supuesta transición ideal perfectamente normalizada las diferencias por razón de sexo, clase social, condiciones económicas, nivel formativo alcanzado, etc. serían evidentes, las transiciones se producen a diferentes ritmos y con distintos niveles de éxito y si aceptamos la idead de unos procesos de transición menos normalizados que en décadas pasadas, las diferencias que se producen serían más acusadas y deben ser estudiadas y puestas en evidencia. En el panorama internacional más allá de las fronteras europeas (Fussell et al., 2007; Sironi et al., 2015; Tian, 2016) en el contexto europeo (Toulemon, 2009; Berrington, 2010; Robette, 2010) y en el panorama español (La Parra, 2000; Vieira y Miret, 2010) son estudios que prestan su atención explícitamente a la heterogeneidad en los procesos de transición a través de diferentes metodologías. En Sironi et al. (2015) se presta especial atención a la clase social de origen para determinar el grado de desestandarización, es decir el grado de distorsión en la secuencia ordinaria de las transiciones, comparando a los Estados Unidos con Italia. En un análisis de regresión la significación estadística entre la clase social de los padres y el grado de desestandarización 
(trabajo antes de terminar los estudios, emancipación muy temprana, emparejamientos antes de experimentar la transición laboral, etc.) es alto en ambos países pero en sentido contrario, mientras en los Estados Unidos entre los jóvenes de las clases altas se produce una mayor desestandarización, en Italia es entre los jóvenes de clases bajas donde se da esta circunstancia. En los países del sur de Europa la emancipación es más temprana entre los jóvenes que proceden de hogares en malas condiciones económicas mientras en el centro y norte de Europa es entre los hogares con mejores condiciones económicas donde los jóvenes se emancipan a edades más tempranas. En el Reino Unido Berrington et al. (2010) revelan las diferencias por sexo como consecuencia de la feminización de la educación superior, las diferencias entre los grupos de edad más jóvenes por sus dificultades en la incorporación al mercado laboral entre 1998 y 2008 y la relativa estabilidad en los eventos de transición entre los jóvenes de mayor edad pero a menudo ocupando múltiples roles combinando por ejemplo estudio y trabajo. En esta década el porcentaje de jóvenes emancipados ha aumentado y la elección de la cohabitación como forma de convivencia en pareja (Berrington et al., 2010). Para Toulemon las secuencias de los eventos de transición siguen siendo válidas a pesar de la creciente desestandarización de la transición, pues permite una descripción global de todo el proceso. Centra su atención en las diferencias por sexo y ha constatado la evolución hacia una disminución de las diferencias entre hombres y mujeres, el retraso en el primer empleo, en la primera unión y en el primer hijo en toda Europa. El matrimonio ya no es la única opción de vida en pareja, consecuentemente aumentan los nacimientos fuera del matrimonio. En la Europa occidental se observa un retraso en la vida en pareja mientras en la Europa del Este no. La transición escolar y laboral se retrasa en todos los países y la emancipación residencial especialmente en los países del Sur de Europa. Las diferencias por sexo aumentan en la Europa del Este y disminuyen en España y Portugal en especial entre las mujeres nacidas en la décadas de los 70 .

Se han citado en este trabajo para el ámbito español frecuentes estudios que hacen referencia a la transición laboral, las dificultades de acceso a empleos adecuados, la precariedad en el trabajo, la falta de linealidad entre formación y empleo, etc. además de las dificultades en la emancipación residencial y el acceso a una vivienda en propiedad o alquiler, pero por separado o incluyendo solamente algunos de los eventos de transición. Los itinerarios de transición en su conjunto no han sido muy tratados y menos aún aquellos que prestan su atención a evidenciar la diferencias o su grado de complejidad, en La Parra (2000) y Vieira y Miret (2010) sí se realizan estas comparaciones sirviéndose de metodologías muy dispares, en el primer caso de entrevistas retrospectivas en profundidad a jóvenes de entre 30 y 34 años que ya han experimentado los eventos de transición o estarían a punto de ello y en el segundo caso sirviéndose del índice de entropía y con datos de censos. En ambos trabajos a pesar de su disparidad en lo que se refiere a la cuestión metodológica, las diferencias se presentan por sexo y en el caso de Vieira y Miret por periodos. Más allá de las conclusiones ya aportadas en otros estudios como el retraso progresivo en todos los procesos de transición y la influencia del contexto familiar aportada por La 
Parra, es necesario evidenciar las carencias observadas en el panorama español en cuanto al estudio de las diferencias entre los jóvenes frente a los procesos de transición en su conjunto y cruzando otras variables además del sexo, como es el riesgo de pobreza o la coyuntura económica. En este artículo se tratará de presentar un enfoque más completo al referirnos al proceso de transición en su conjunto, aportando información relativa a los cinco apartados mencionados y observando el grado de heterogeneidad que hay en el colectivo objeto de estudio teniendo en cuenta variables independientes como el sexo, periodo de coyuntura económica y riesgo de pobreza.

\section{DATOS Y MÉTODO}

El objetivo perseguido será el valorar la heterogeneidad de cada grupo de edad según el grado de complejidad de todas las posibles combinaciones de los cinco estatus o estados que representarían cada transición (estudiante, activo, emancipado, vida en pareja y padre) medido con el índice de entropía. Además de la valoración de la heterogeneidad de cada grupo de edad y cruzando las variables independientes habituales, será posible construir secuencias de transición donde poder identificar el término de la transición hacia la adultez. Estos objetivos conducirán a refutar la hipótesis, que es posible plantear una vez expuesto el apoyo teórico, según la cual los itinerarios que conducen a la transición hacia la vida adulta son diversos y heterogéneos e influenciados por la estructura económica, la estructura de clase heredada de los padres y las variables sociodemográficas que influyen en la diversidad de itinerarios y tienden a la segmentación social de los jóvenes.

\section{Bases de datos}

La ECV (Encuesta de Condiciones de vida) es la base de datos utilizada para la elaboración de los indicadores que se presenta en adelante. Esta encuesta aporta una gran información, especialmente sobre el nivel renta, pobreza y exclusión social, si bien, por su magnitud cuantitativa, su alto grado de calidad en lo que a su actualidad y grado de comparabilidad se refiere, la inclusión de multitud de otro tipo de variables, como son las demográficas, etc., permite el análisis de otros muchos aspectos de la realidad social. El INE (Instituto Nacional de Estadística) ofrece los microdatos en formato CSV (formato de texto separado por comas). Se han fusionado los archivos de los años 2006 y 2007, que representan el momento previo a la crisis económica, y los años 2014 y 2015 como momento en el que se comienzan a observar indicadores que reflejan la salida de la crisis. El motivo de la fusión ha sido el trabajar con ficheros con un número de casos mayor, con el objetivo de minimizar la distorsión en los resultados que se puede producir por la escasez de casos para algunas de las categorías de las distribuciones con las que se ha trabajado.

La encuesta ofrece casos a partir de los 17 años y para la construcción de secuencias de transición con los índices de entropía se ha decidido seleccionar la 
edad hasta los 46 años, con el propósito de visualizar más claramente el término de la transición, no obstante los indicadores únicamente se presentan por grupos de edad de tres y se analiza la información obtenida hasta los 34 años.

\section{Variables seleccionadas}

De entre las numerosas variables que ofrece la ECV se han seleccionado aquellas que más directamente influyen en los procesos de transición, algunas de ellas tal y como las ofrece la encuesta y otras modificadas e incluso creadas a partir de las que la encuesta incluye. Las variables dependientes son las siguientes:

Estar o no estar estudiando, esta variable la ofrece tal cual la ECV.

Ser o no ser activo, esta variable no la ofrece la encuesta, si bien se ha conseguido mediante una agrupación de las diferentes categorías de activos y de no activos que contiene la variable relación con la actividad.

Estar o no estar emancipado, esta variable no está incluida en la ECV, sumando a los que tienen cónyuge o pareja de hecho (se ha supuesto que los que tienen cónyuge o viven en pareja, con independencia del tipo de unión, estarían todos emancipados) más aquellos que no tienen cónyuge o pareja de hecho, pero son responsables de hogar.

Tener o no tener cónyuge o pareja de hecho, esta variable la aporta la encuesta, pero con tres categorías, sí con base jurídica, sí sin base jurídica y no, agrupando las dos primeras categorías se dispone de la variable dicotómica buscada. La categoría en positivo incluiría a todas las personas con relación de pareja y que viven en el mismo hogar, con independencia del modelo de pareja; estarían incluidos matrimonios, parejas de hecho o cualquier otro tipo de relación de pareja con independencia de que exista o no base jurídica. Para evitar confusiones, se ha decidido nombrar la nueva variable con el nombre de vida en pareja y las categorías sí y no, y la transición a la que se accedería transición a vida en pareja. De tal manera que siempre que se esté haciendo referencia a pareja se estará refiriendo a parejas que cohabitan, en ningún caso relaciones de noviazgo u otro tipo de relación que no implique compartir el mismo hogar.

Ser o no ser padre, esta variable no se incluye en la encuesta y se ha obtenido sumando a los que viven en pareja y en hogares con hijos, a los responsables de hogar que viven en hogares con hijos y sin pareja. No es un dato exacto, pero sí muy aproximado, pues parece lógico pensar que todos aquellos que viven en hogares con hijos y son pareja son padres, como también lo son aquellos responsables de hogar sin pareja, pero que viven en hogares con hijos.

Como variables independientes se han utilizado: la variable edad, la variable sexo y la variable riesgo de pobreza del hogar, que la ECV ofrece con las categorías sí o no. La coyuntura económica, representada por los años 2006-2007, antes de la crisis, y en la salida de la crisis 2014-2015 aportará el análisis comparativo buscado. 


\section{Análisis de entropía}

Las posibilidades de aplicación del análisis de entropía en ciencias sociales han sido exploradas por diversos autores, fundamentalmente para los análisis de la desigualdad de la renta (Dagum, 1993; Lechuga, 1998). Una aplicación menos estudiada es para la que se utiliza este indicador en el presente trabajo, valorar la heterogeneidad de la juventud en los procesos de transición. En Vieira (2013) se ponen de manifiesto estas posibilidades recordando que el concepto de entropía se desarrolla originalmente en la física, en estudios para medir la transformación de la materia en un sistema determinado; la entropía mediría el grado de segmentación o fragmentación dentro del sistema. En este sentido, la entropía es una medida de descomposición y también de heterogeneidad, pues cuanto mayor es la entropía, mayor será la complejidad de un fenómeno. Vieira explica el procedimiento matemático a seguir en el uso de este indicador en ciencias sociales y basa sus explicaciones en los análisis estadísticos de descomposición propuestos por Theil (1972); por tanto, el índice de entropía es un indicador que puede ser utilizado para valorar la heterogeneidad de los jóvenes en la transición a la vida adulta según la mayor o menor complejidad en una distribución de posibles combinaciones de estatus ${ }^{1}$ (Fussell et al., 2007; Vieira y Miret, 2010; Vieira, 2013; Felicia F. Tian, 2016). En este trabajo, la entropía se utilizará para medir la heterogeneidad de un colectivo midiendo la complejidad en las distribuciones compuestas por las posibles combinaciones de los estatus o estados que definen la transición hacia la adultez. Estos estatus se producen en diferentes momentos y en ocasiones se solapan, de tal manera que la combinación de los diferentes estatus es distinta según el momento vital en el que el joven se encuentre. Así, es posible estar formándose, incorporarse al mercado laboral, tener pareja y emanciparse, como también es posible únicamente estar formándose y no asumir ninguno del resto de estados. Los cambios y ajustes en la transición hacia la vida adulta hacen pensar en un sistema donde intervienen diferentes elementos, el sistema sería el proceso de transición en su conjunto y los elementos que entran y salen del sistema las diferentes combinaciones de estatus que se asumen en cada momento del ciclo vital; el índice de entropía mide la complejidad en la combinación de estos estatus para cada edad, periodo, sexo y demás variables consideradas. La mayor complejidad en la distribución de estatus representada por el punto ápice de entropía correspondería con el final del periodo de transición, mientras que la disminución y la estabilidad indicarían la culminación de la transición hacia la vida adulta (Vieira, 2013). En una combinación donde intervengan los estatus de estudiante, activo, emancipado, con pareja y ser padre, todos los individuos de 12 años, por ejemplo, se con-

${ }^{1}$ La bibliografía consultada que sirve de apoyo en la elaboración del análisis de entropía utiliza la terminología de estatus para referirse a la situación en la que se encuentra el joven con relación a los estudios, el mercado laboral, la emancipación, etc., si bien la palabra estatus utilizada estrictamente haría referencia a la posición en la estructura social. Con la intención de no crear confusión, se ha decidido utilizar indistintamente la palabra estatus y estado para referirse a la situación en la que se encuentra un joven con relación a los estudios, el mercado laboral, la emancipación residencial, la vida en pareja o a ser padres. 
centrarían en una posible combinación estudiantes, no activos, no emancipados, sin pareja y sin hijos; en este ejemplo la entropía sería 0; las combinaciones de estatus o estados se van complicando progresivamente al compartir diferentes estatus y cambiar con frecuencia esta combinación, estaríamos hablando de los años centrales de la juventud; la máxima entropía posible depende del número de estatus que se combinen y se produciría en aquella distribución donde para cada una de sus categorías existe el mismo número de casos. La etapa del ciclo vital en la que las personas asumen posiciones muy similares: la mayor parte no estudian, son activos, están emancipados residencialmente, tienen pareja y tienen hijos, se corresponde con una etapa del ciclo vital donde la entropía o heterogeneidad tiende a estabilizarse.

A través del índice de entropía será posible describir la trayectoria hacia la adultez en función del grado de complejidad en la distribución de la combinación de estatus que proporciona el índice de entropía. Para poder valorar las diferencias en el proceso de transición se realizarán diferentes comparaciones: en dos momentos de diferente coyuntura económica, diferencias por sexo y diferencias cuando se pertenece a un hogar con o sin riesgo de pobreza.

Descripción del indicador

Todo el razonamiento metodológico está basado en el uso del índice de entropía general de Theil y en las indicaciones propuestas por Vieira, Miret y Fussell. La fórmula matemática es la siguiente:

$$
E_{x}=\sum_{s} p_{s, x} \cdot \ln \left(1 / p_{s, x}\right)
$$

Donde $E$ es el índice de entropía, $s$ indica una determinada combinación de estatus, $x$ a una cierta edad y $P_{s x}$ la proporción de la población $x$ que se encuentra en el estatus $s$. El índice de entropía es el sumatorio de la proporción de una edad determinada que se encuentra en la combinación de estatus multiplicado por el logaritmo natural de la proporción inversa de esa combinación.

El índice de entropía va desde el 0, cuando la homogeneidad es perfecta, es decir, cuando todos los individuos están concentrados en el mismo estatus o combinación de estatus, hasta la entropía máxima, que varía dependiendo del número de posibles combinaciones de estatus y se refiere a la máxima heterogeneidad, cuando existe el mismo número de casos para cada combinación de estatus. En Fussell (2005) se recomienda convertir los índices en porcentajes para hacer más entendible la información que se presenta; para conseguir este fin ha sido necesario calcular la entropía máxima, siendo la entropía máxima el 100\% resulta simple calcular el porcentaje que corresponde a la entropía obtenida. La representación gráfica permite visualizar claramente la evolución de la entropía facilitando los análisis y la comprensión de los resultados (Vieira y Miret, 2010). La entropía máxima está definida por la siguiente fórmula:

$$
E_{\max }=\Sigma\left(1 / C_{s}\right) \cdot \ln \left[1 /\left(1 / C_{s}\right)\right]
$$


Donde $E_{m a x}$ es la entropía máxima y $C_{s}$ es el número total de combinaciones de estatus posibles. La entropía máxima para la combinación de cinco estatus es de 3,466 teniendo en cuenta que el número total de combinaciones posibles es de 32 .

\section{ANÁLISIS Y RESULTADOS}

En el gráfico 1 se presenta el porcentaje de entropía antes de la crisis económica y en la salida de la crisis económica. La primera de las lecturas que es posible realizar de los indicadores representados en este gráfico correspondería al aumento progresivo del índice de entropía conforme aumenta la edad; es la consecuencia lógica de que la juventud va asumiendo más complejidad en la combinación de estatus, hasta el ápice de entropía, que como se ha dicho representa el momento en el que terminaría la transición y se producirá la incorporación a la adultez. El ápice de entropía para el periodo precrisis está bien definido en la cohorte de 29-31 años, pero no es tan evidente para el periodo postcrisis, donde se produce una continuación del mismo índice entre esta cohorte y los 3234 años. Aunque no con total claridad, sí es posible interpretar un retraso en la transición en este periodo con relación al anterior. La observación más evidente son las diferencias entre los dos periodos y en las cohortes de entre quienes tiene menor edad, 17-19 y 20-22 años, con un porcentaje de entropía para los primeros del $25 \%$ y $17 \%$ en precrisis y postcrisis, respectivamente, y del $37 \%$ y $32 \%$ para la cohorte de 20-22 años e igualmente en precrisis y postcrisis, respectivamente. La mayor entropía entre quienes tienen menor edad en el periodo precrisis permitiría deducir que en una buena coyuntura económica hay mayor facilidad de incorporación al mercado laboral y que la juventud comienza antes a combinar diferentes estados y, por tanto, a aumentar el grado de heterogeneidad. A partir de los 23-25 años aumenta el porcentaje de entropía progresivamente, pero no existen diferencias entre las cohortes de los dos periodos de referencia, salvo lo comentado con relación al ápice de cada secuencia, que haría pensar en un cierto retraso en la transición en el periodo que corresponde con la salida de la crisis económica. 


\section{Gráfico 1. Porcentaje de entropía antes de la crisis económica y en la salida de la crisis.}

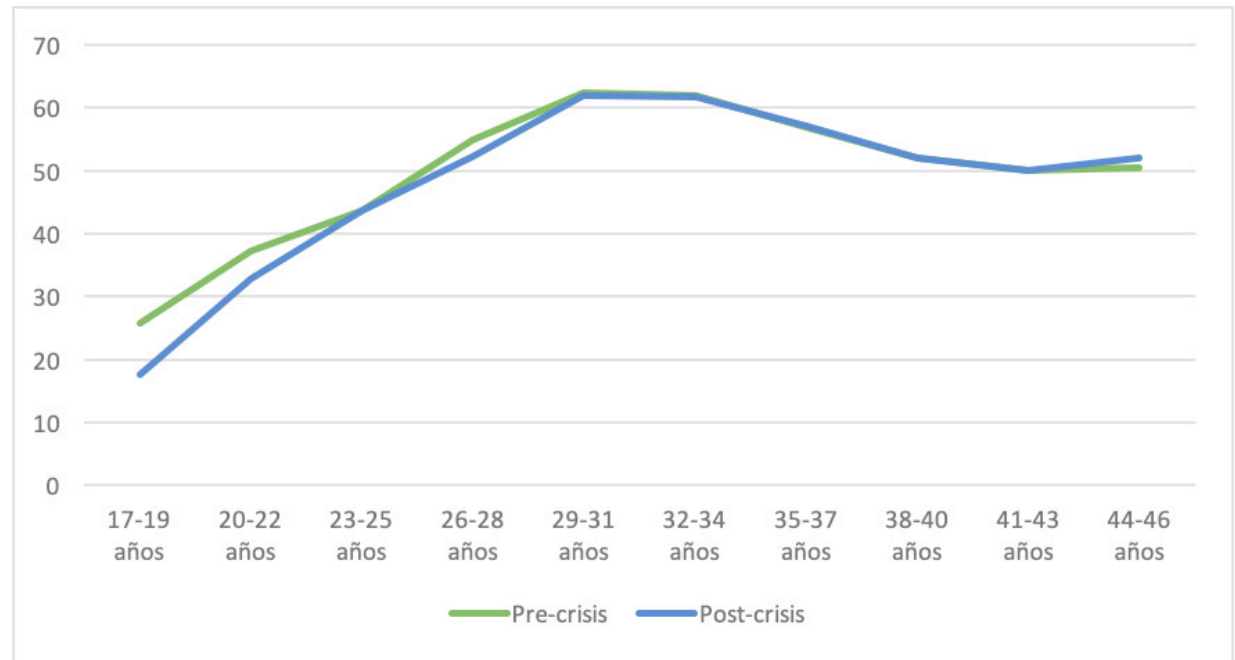

Fuente: elaboración propia con datos de la ECV.

Diferencias por sexo. Los varones, más tiempo jóvenes que las mujeres

En el gráfico 2 se presenta el índice de entropía para los dos periodos de referencia y por sexo. La primera lectura que es posible realizar sobre las secuencias que describe la representación gráfica es la diferencia por sexo en todas las cohortes menos en la de menor edad, 17-19 años, para la cual las diferencias que se observan son por coyuntura económica y no por sexo; tanto varones como mujeres tendrían un porcentaje de entropía de en torno al $25 \%$ en el periodo previo a la crisis económica y del $17,5 \%$ en el periodo que se ha considerado como el de la salida de la crisis económica. A partir de los 20-22 años, los varones no presentan diferencias entre los dos periodos y el ápice de entropía se sitúa en ambos periodos en el intervalo de 32-34 años con un 55\% de entropía en precrisis y un $58 \%$ en postcrisis. Para las mujeres, las diferencias por coyuntura económica se extienden hasta los 23-25 años y el ápice de entropía se sitúa a los 29-31 años, con un porcentaje de entropía del 66\% para ambos periodos. Lo más relevante del análisis de estos datos está en las diferencias por sexo más que por coyuntura económica, con diferencias por encima de 10 puntos porcentuales más en mujeres que en varones, con la excepción de las cohortes más jóvenes. La diferencia en el ápice de entropía sería de una cohorte antes en las mujeres que en los varones. En resumen, en las mujeres se produce una mayor complejidad en la distribución de estatus en toda la transición hacia la adultez y esta se completaría tres años antes que en los varones, no observándose diferencias por coyuntura 
económica, salvo en las cohortes de menor edad y en todo caso disminuyendo ligeramente la entropía en postcris.

\section{Gráfico 2. Porcentaje de entropía en el periodo precrisis y postcrisis por sexo.}

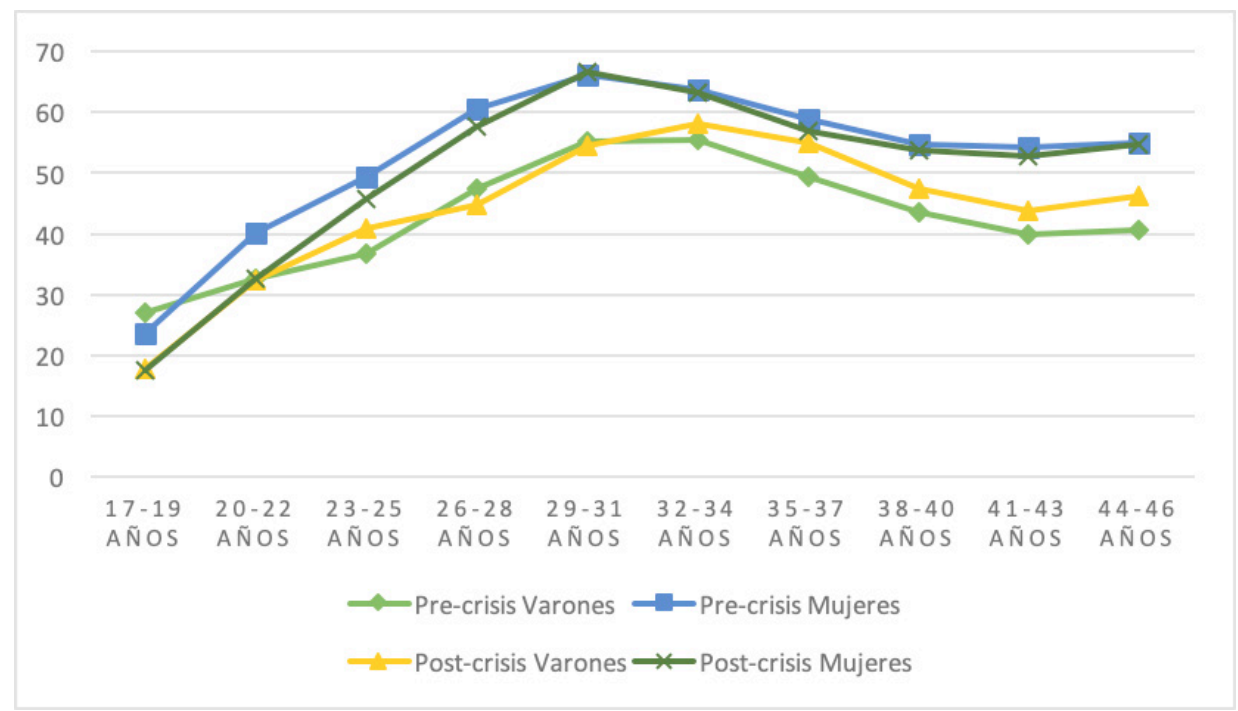

Fuente: elaboración propia con datos de la ECV.

\section{El riesgo de pobreza y las diferencias en la transición hacia la adultez}

En el gráfico 3 se presentan los índices de entropía y las secuencias de transición para los hogares en riesgo y sin riesgo de pobreza en el periodo anterior a la crisis y en el de la salida de la crisis económica. La primera lectura que es posible realizar es el retraso en la salida de la transición entre los que pertenecen a hogares con buenas condiciones económicas frente a aquellos en riesgo de pobreza; la lectura es posible hacerla para ambos periodos, estando el ápice de heterogeneidad entre quienes menos recursos tienen en su hogar de origen a los 29-31 años, frente a los 32-34 cuando en los hogares a los que se pertenece tienen mejores condiciones económicas, con un $64 \%$ y $61 \%$, respectivamente. La entropía es mayor en aquellos que pertenecen a hogares en riesgo de pobreza, con algunas diferencias por edad y periodo; en este sentido, entre los que pertenecen a hogares en riesgo de pobreza las diferencias entre periodos son pequeñas, entre 3 y 4 puntos porcentuales y únicamente entre las cohortes de menor edad, 17-25 años. Entre quienes pertenecen a hogares sin riesgo de pobreza las diferencias son mayores, entre 8 y 12 puntos, pero únicamente en las dos cohortes de los de menor edad, 17-19 y 20-22 años. En resumen, las diferencias no estarían tanto entre periodos, estarían especialmente influenciadas por el riesgo 
de pobreza, con una mayor heterogeneidad entre quienes son más pobres y un periodo de transición de una cohorte menos.

\section{Gráfico 3. Porcentaje de entropía en el periodo precrisis y postcrisis según se pertenezca a un hogar en riesgo o no de pobreza.}

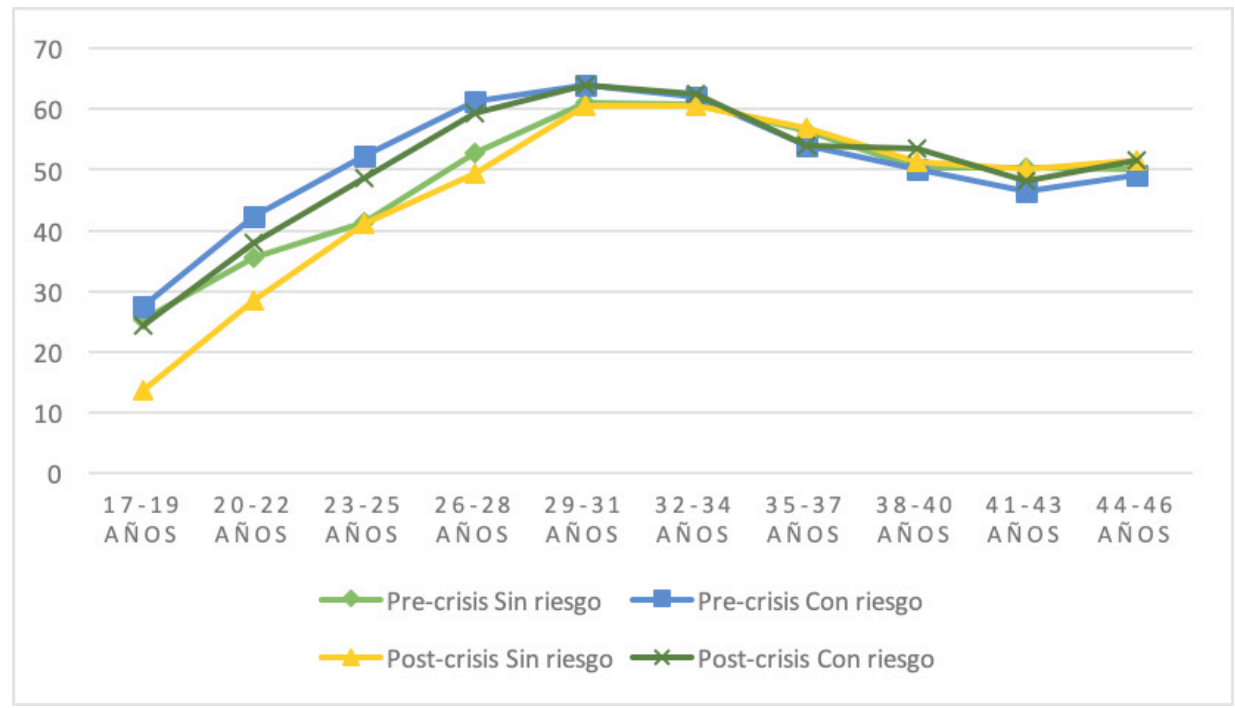

Fuente: elaboración propia con datos de la ECV.

En los gráficos 4 y 5 se ofrecen los índices de entropía para varones y mujeres, respectivamente, por riesgo de pobreza y en los periodos precrisis y postcrisis. Se advirtió en el apartado metodológico que la elección hasta los 46 años de edad está motivada únicamente con el fin de visualizar con mayor claridad la secuencia de transición, por tanto, lo que sucede en las cohortes por encima de la edad de 34 años excede del análisis que se requiere para este trabajo. Ciertamente, puede suscitar interés la diferencia de entropía a partir de los 34 años de edad entre los periodos de precrisis y postcrisis entre los varones, pero no es una cuestión a valorar en este trabajo de investigación. Lo más destacado para el gráfico que corresponde a los varones es que las diferencias no son tanto por periodo como por riesgo de pobreza, con la excepción del grupo de 17-19 años en postcrisis y sin riesgo de pobreza, en el que disminuye la entropía entre los dos periodos en torno a 15 puntos porcentuales menos de entropía en postcrisis. También se puede observar una diferencia importante, 10 puntos porcentuales, en el grupo de 32-34 años entre aquellos que sufren riesgo de pobreza en el sentido de más entropía en el periodo de postcrisis. Excluyendo estas dos excepciones, las diferencias en la heterogeneidad en el proceso de transición son únicamente por pertenecer o no a un hogar en riesgo de pobreza, con un mayor índice de entropía entre los que sufren este riesgo y un adelanto en completar 
la transición de una cohorte con relación a aquellos que no sufren el riesgo de pobreza; al igual que en el apartado anterior, cuando se han considerado ambos sexos en conjunto, aquellos que sufren riesgo de pobreza culminarían el proceso de transición a los 29-31 años, mientras aquellos varones que no tienen este riesgo lo culminan a los 32-34 años. Para el caso de las mujeres, igualmente, las diferencias más importantes se producen por riesgo de pobreza, si bien en este caso también es posible observar una disminución en el índice de entropía en el periodo de postcrisis tanto en aquellas que tienen riesgo de pobreza como en aquellas que no lo tienen. Lo más significativo es que las mujeres presentan un mayor índice de entropía que los varones en todas las comparaciones y que la edad a la que se produce el ápice de entropía se adelanta una cohorte cuando las mujeres sufren riesgo de pobreza con relación a cuando se ha realizado la comparación por sexo, pero sin tener en cuenta esta variable. Anteriormente, se ha observado cómo el ápice de entropía en las mujeres se producía a los 29-31 años y a los 32-34 años en los varones, se sigue produciendo el ápice de entropía a los 29-31 años en ambos periodos de referencia y para las mujeres que no presentan riesgo de pobreza, pero se adelanta una cohorte en aquellas mujeres que sufren riesgo de pobreza, tanto en precrisis como en postcrisis, hasta los 26-28 años. Nuevamente, las diferencias por sexo, junto al riesgo de pobreza, son los elementos que más influencia tienen en la heterogeneidad de los jóvenes en sus procesos de transición. Las mujeres presentan una mayor heterogeneidad en la asunción de estatus, lo que indica que simultáneamente están asumiendo un mayor número de roles o estatus con relación a los varones, culminan su transición antes que estos y, si son pobres, se produce un adelanto extra; la feminización de la pobreza, con peores posibilidades de acceso al mercado laboral, junto a motivos culturales que acentúan la división de roles entre varones y mujeres, asociando ocupaciones de mayor prestigio y recursos económicos a los varones frente a las mujeres, contribuyen a acentuar las dificultades económicas entre las mujeres. 
Grafico 4. Entropía en varones en pre y post crisis y riesgo de pobreza.

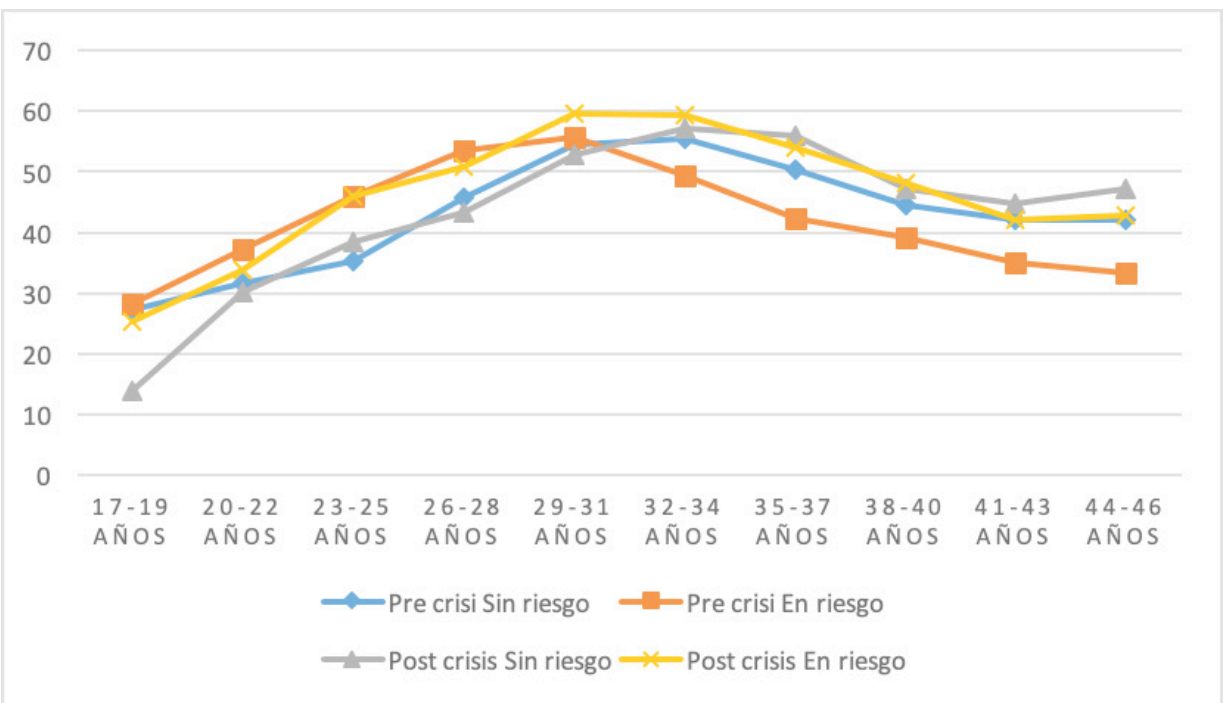

Fuente: Elaboración propia con datos de la ECV años 2006-2007-2014-2015.

Grafico 5. Entropía en mujeres en pre y post crisis riesgo de pobreza.

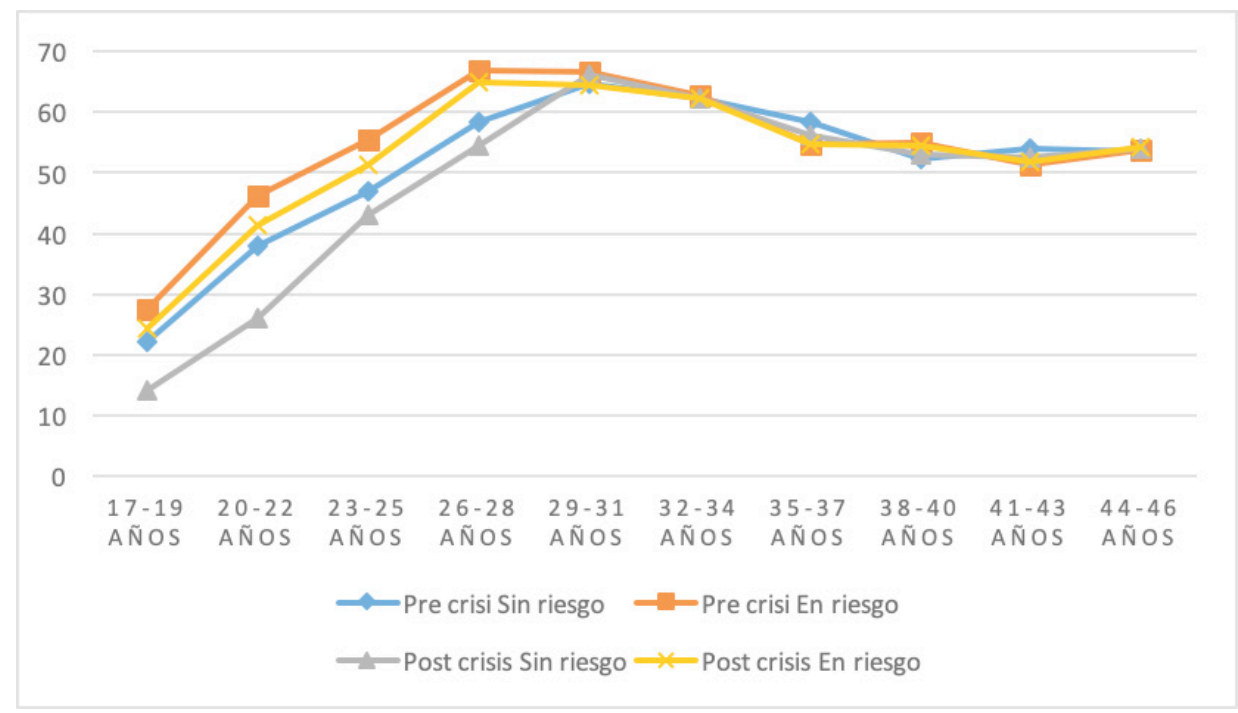

Fuente: Elaboración propia con datos de la ECV años 2006-2007-2014-2015. 


\section{CONCLUSIONES}

La posibilidad del índice de entropía en la medida de la heterogeneidad en la juventud con relación a la simultaneidad en la asunción de los roles de adultos, queda explicitada en los indicadores que se han ofrecido en el análisis de resultados anterior. En este sentido, la heterogeneidad aumenta progresivamente desde las cohortes más jóvenes hasta las de mayor edad, las mujeres presentan mayores índices de entropía, es decir, de heterogeneidad que los varones, adelantando tres años la salida hacia adultez. Sería posible decir que las mujeres son jóvenes durante menos tiempo que los varones, comienzan su transición en el mismo momento, pero más intensamente que los varones y terminan en torno a tres años antes que estos. Por diferente coyuntura económica, se observa una disminución de la heterogeneidad entre los más jóvenes motivada por las dificultades en el acceso al mercado laboral, lo que repercute en que más jóvenes se encuentren estudiando y no trabajando; aunque los datos no son concluyentes, se observa un cierto alargamiento en la transición hacia la adultez en el periodo que corresponde con la salida de la crisis respecto del periodo previo a la crisis.

Se produce un retraso en la salida de la transición entre los que pertenecen a hogares con buenas condiciones económicas frente a aquellos en riesgo de pobreza; la lectura es posible hacerla para ambos periodos, estando el ápice de heterogeneidad (que correspondería con el final de la transición) entre quienes menos recursos tienen en su hogar de origen a los 29-31 años, frente a los 32-34 cuando en los hogares a los que se pertenece tienen mejores condiciones económicas. La entropía es mayor en aquellos que pertenecen a hogares en riesgo de pobreza, con algunas diferencias por edad y periodo; en este sentido, entre los que pertenecen a hogares en riesgo de pobreza las diferencias entre periodos son pequeñas, entre 3 y 4 puntos porcentuales y únicamente entre las cohortes de menor edad, 17-25 años. Entre quienes pertenecen a hogares sin riesgo de pobreza las diferencias son mayores, entre 8 y 12 puntos, pero únicamente en las dos cohortes de los de menor edad: 17-19 y 20-22 años. Las diferencias nuevamente no estarían tanto entre periodos, estarían especialmente influenciadas por el riesgo de pobreza, con una mayor heterogeneidad entre quienes son más pobres y un periodo de transición de una cohorte menos.

Sería posible concluir diciendo que la crisis económica no habría provocado cambios sustanciales en lo que se refiere a una mayor complejidad y diversidad en la transición hacia la adultez. Únicamente es en los intervalos de menor edad donde se observan diferencias por coyuntura económica que estarían claramente relacionados con la dificultad de acceso en estos grupos al mercado laboral en tiempos de crisis, lo que provoca una prolongación en el pedido formativo disminuyendo el índice de entropía. Con la excepción de los grupos de menor edad, en las mujeres se observa una mayor complejidad en el proceso de transición, pero atenuándose estas diferencias en el periodo de crisis económica. Como se ha dicho las mujeres completan tres años antes que los varones la transición y si proceden de hogares en riesgo de pobreza tres años más. Sería posible establecer cierta analogía entre ser mujer y ser pobre, en ambos casos las transición se com- 
pleta antes que si se es varón y sin riesgo de pobreza. O dicho de otra manera, ser mujer o pertenecer a un hogar en riesgo de pobreza acortan el periodo de la juventud.

\section{REFERENCIAS}

Aassve, Arnstein, María A. Davia, María Iacovou, y Letizia Mencarini (2005). Poverty and the transition to adulthood: risky situations and risky events (23). ISER Working Paper Series.

Beck, Ulrich. (1998). La sociedad del riesgo: hacia una nueva modernidad. Barcelona: Paidós.

Becker, Gary (2005). Inversión capital humano e ingresos. En L. Toharia (Ed.), El mercado de trabajo: Teorías y aplicaciones. Madrid: Alianza.

Berrington, Ann, Juliet Stone y Jane Falkingham (2010). How far have transitions to adulthood changed in the last decade? Evidence from the United Kingdom Labour Force Survey. Centre for Population Change Working Paper 5.

Bird, Katherine y Helga Krüger (2005). The secret of transitions: The interplay of complexity and reduction in life course analysis. Advances in life course research, (10), 173-194.

Carbajo, Diego (2014). Vivir en la precariedad. Trayectorias y estrategias residenciales de la juventud en la Comunidad Autónoma del País Vasco. Tesis doctoral. Directores: Benjamín Tejerina y Iñaki Martinez, Universidad del País Vasco. Recuperada el día 5/7/2017 en https:// addi.ehu.es/bitstream/handle/10810/18295/9082-414-6-CarbajoTH. pdf? sequence $=1$

Casal, Joaquin, Maribel García, Rafael Merino y Miguel Quesada (2006) Aportaciones teóricas y metodológicas a la sociología de la juventud desde la perspectiva de la transicion. Revista de Sociología. Universidad Autónoma de Barcelona, (79), 21-48.

Castells, Mauel (1997). La era de la información. Economía, sociedad y cultura, 3 vols. Madrid: Alianza Editorial. [1997, vol. 1; 1998, vols. 2 y 3$]$.

Dagum, Camilo (1993). Fundamentos de Bienestar Social de las medidas desigualdad en la distribución de la renta. Cuadernos de Ciencias Económicas y Empresariales, (24), 11-36.

Du Bois-Reymond, Manuela y Andreu López Blasco (2004). Transiciones tipo yo-yo y trayectorias fallidas: hacia las políticas integradas de transición para los jóvenes europeos. Revista de Estudios de Juventud, 65(04), 11-29.

Fahmy, Eldin (2007). Poverty and Youth Transitions in Europe: An Analysis of the European Communities Household Panel. En H. Colley, P. Boetzelen, B. Hoskins y T. Parveva (Eds.), Social inclusion for young people: breaking down the barriers. Council of Europe Publishing.

Furstenberg, Frank F. (2005). Non-normative life course transitions: reflections on the significance of demographic events on lives. Advances in Life Course Research, (10), 155-172. 
Fussell, Elizabeth (2005): Measuring the early adult life course in Mexico: an application of the entropy index, Advances in Life Course Research, 9: 91-122.

Fussell, Elizabeth, Anne H. Gauthier y Ann Evans (2007). Heterogeneity in the transition to adulthood: The cases of Australia, Canada, and the United States. European Journal of Population/Revue Européenne de Démographie, 23 (3-4), 389-414.

Gaviria, Sandra (2017). El retorno al hogar de los padres en las diferentes etapas de la vida en Francia. METAMORFOSIS, 2017, p. 7-19.

Gentile, Alessandro (2010). De vuelta al nido en tiempos de crisis. Los boomerang kids españoles. Revista de estudios de juventud, 90(10), 181-203.

Gentile, Alessandro (2016). La emancipación juvenil en tiempos de crisis: un diagnóstico para impulsar la inserción laboral y la transición residencial. METAMORFOSIS, 119-124.

Giddens, Anthony y Joaquín Bollo Muro (1979). La estructura de clases en las sociedades avanzadas. Madrid: Alianza, pp. 202, 313, 341.

Iacovou, María (2011). Leaving home: independence, togetherness and income in Europe. United Nations, Department of Economic and Social Affairs. Population Division.

La Parra Casado, Daniel (2000). Desigualdades de género durante la transición a la vida adulta. Papers: revista de sociología, (61), 113-124.

Leccardi, Carmen (2010). La juventud, el cambio social y la familia: De una cultura "de protección" a una cultura "de negociación". Revista de Estudios de Juventud, (90), 33-42.

Lechuga, Matilde L. (1998). La distribución Gamma como modelo para analizar la distribución de la renta: Una aplicación a la EPF 19901991. Revista de Estudios Regionales, (50), 161-186

Moreno, Almudena, Antonio López y Sagrario Segado (2012). La transición de los jóvenes a la edad adulta. Crisis económica y emancipación tardía. Barcelona: Obra Social La Caixa.

Robette, Nicolás (2010). The diversity of pathways to adulthood in France: Evidence from a holistic approach. Advances in Life Course Research, 15 (2), 89-96.

Sánchez-Galán, F. Javier y Moreno, Almudena (2017). Las instituciones sociales y la desigualdad entre los jóvenes. Nivel formativo, tipo de ocupación y reproducción intergeneracional como elementos diferenciadores. Comunitania. Revista Internacional de Trabajo Social y Ciencias Sociales, (13), 179-209.

Santamaría López, Elsa (2012). Jóvenes y precariedad laboral: trayectorias laborales por los márgenes del empleo. Zerbitzuan: Gizarte zerbitzuetarako aldizkaria = Revista de servicios sociales, (52), 129-139.

Sironi, María, Nicola Barban y Roberto Impicciatore (2015). Parental social class and the transition to adulthood in Italy and the United States. Advances in Life Course Research, (26), 89-104.

Tezanos, Jose Felix (2001). El trabajo perdido: ¿hacia una civilización postlaboral? Madrid: Biblioteca Nueva. 
Theil, Henry (1972). Statistical decomposition analysis; with applications in the social and administrative sciences. Amsterdam: North Holland Publ.

Tian, Felicita (2016). Transition to adulthood in China in 1982-2005: a structural view. Demographic Research, 34, 451

Torres, Anália y Tiago Lapa (2010). Familia y jóvenes en Europa. Convergencia y diversidad. Revista de Estudios de Juventud, (90), 11-32.

Toulemon, Laurent (2009). Transition to adulthood in Europe: Is there convergence between countries and between men and women? European Commission Directorate-General Employment, Social Affairs and Equal Opportunities. Unit E1 - Social and Demographic Analysis, December 2009.

Ulrich, Karl (2008). New Directions in Life Course Research. Yale University, Center for Research on Inequalities and the Life Course. ciqle@yale.edu www.yale.edu/ciqle

Urcola, Marcos (2003). Algunas apreciaciones sobre el concepto sociológico de juventud. Invenio, 6 (11), 41-51.

Vieira, Joice Melo (2013). Possibilidades de aplicação da análise de entropia nas Ciências Sociais e na Demografia. Idéias-Revista do Instituto de Filosofia e Ciências Humanas da UNICAMP.

Vieira, Joice y Pau Miret (2010). Transición a la vida adulta en España: una comparación en el tiempo y en el territorio utilizando el análisis de entropía. Reis: Revista Española de Investigaciones Sociológicas, (131), 75-107. 